FISSION, FUSION AND ANNIHILATION IN

THE INTERACTION OF LOCALIZED STRUCTURES FOR

THE (2+1)-DIMENSIONAL GENERALIZED BROER-KAUP SYSTEM

By

Emmanuel Yomba

and

Yan-ze Peng

IMA Preprint Series \# 2050

(May 2005)

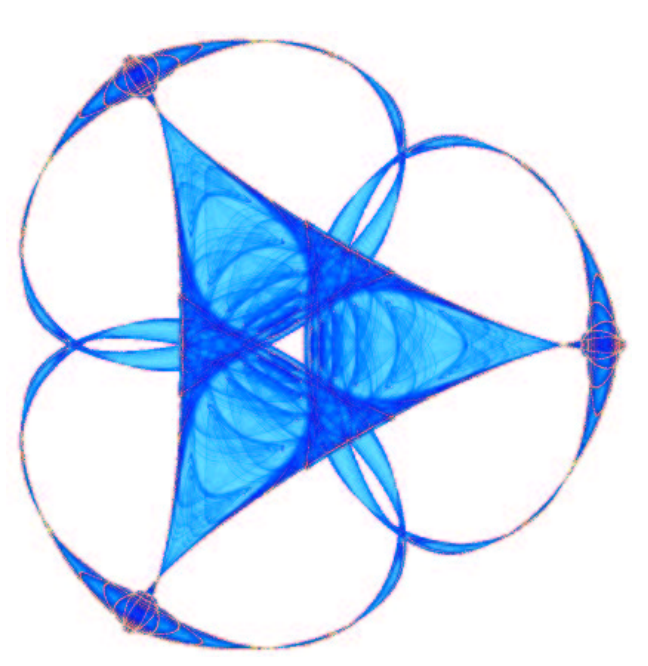

INSTITUTE FOR MATHEMATICS AND ITS APPLICATIONS

UNIVERSITY OF MINNESOTA 400 Lind Hall

207 Church Street S.E.

Minneapolis, Minnesota 55455-0436

Phone: 612/624-6066 Fax: 612/626-7370

URL: http://www.ima.umn.edu 


\title{
Fission, Fusion and Annihilation in the Interaction of Localized Structures for the (2+1)-dimensional Generalized Broer-Kaup System
}

\author{
Emmanuel Yomba ${ }^{a, b}$, Yan-ze Peng ${ }^{c}$
}

${ }^{a}$ Institute for Mathematics and its applications, University of Minnesota, 400 Lind Hall 207 Church Street S.E. Minneapolis, MN 55455 U.S.A.

${ }^{b}$ Department of Physics, Faculty of Sciences, University of Ngaoundéré PO. BOX 454 Ngaoundéré Cameroon.

${ }^{c}$ Department of Mathematics, Huazhong University of Science and Technology, Wuhan 430074, PR China.

\section{ABSTRACT}

Based on the WTC truncation method and the general variable separation approach (GVSA), we have first found a general solution including three arbitrary functions for the (2+1)-dimensional simplified generalized Broer-Kaup (GBK) system $(B=0)$. A class of double periodic wave solutions is obtained by selecting these arbitrary functions appropriately. The interaction properties of the periodic waves are numerically studied and found to be non-elastic. Limit cases are considered and some new localized coherent structures are obtained, the interaction properties of these solutions reveal that some of them are completely elastic and some are non completely elastic. After that, starting from the $(2+1)$-dimensional GBK system $(B \neq 0)$ and using the variable separation approach (VSA) including two arbitrary functions in the general solution, we have constructed by selecting the two arbitrary functions appropriately a rich variety of new coherent structures. The interaction properties of these structures reveal new physical properties like fusion, fission, or both and present mutual annihilation of these solutions as time increasing. The annihilation in this model has found to be rule by the parameter $K_{1}$, when this parameter is taken to be zero, the annihilation disappears in this model and the above mentioned structures recover the solitonic structure properties. 


\section{INTRODUCTION}

Early in the study of soliton theory, the main interests of scientists were restricted to the (1+1)-dimensional cases because of the difficulty of finding the physically significant high-dimensional solutions which are localized in all directions. Today, it is found that the study of the $(1+1)$-dimensional solitons is reaching a certain stage of maturity, the soliton structures and properties of the (1+1)-dimensional integrable nonlinear partial differential equations (NLPDEs) have been now understood very well. However, the study of higherdimensional soliton effects is still in its infancy, the soliton structures in higher spatial dimensions continue to be much more intricate and to attract much more attention. To find some accurate localized coherent soliton solutions in case of $(2+1)$-dimensions is very important and physically significant because many $(2+1)$-dimensional NLPDEs are obtained from the physical systems. Thus, other important problem in nonlinear science is to extend lower dimensional integrable models to higher dimensional ones which are very close to the real natural phenomena without lost their integrability.

In Ref. [1], Zhang and co-workers investigated the (1+1)-dimensional Broer-Kaup system $[2,3]$

$$
\left\{\begin{array}{l}
u_{t}=u u_{x}+v_{x}-\frac{1}{2} u_{x x}, \\
v_{t}=(u v)_{x}+\frac{1}{2} v_{x x},
\end{array}\right.
$$

Which describes the bi-directional propagation of long waves in shallow water. Then taking into account the Painlevé Property (leading order analysis, resonant points determination and the resonance condition verifications), they derived the following (2+1)-dimensional generalized Broer-Kaup (GBK) system

$$
\left\{\begin{array}{l}
H_{t}-H_{x x}-2 H H_{x}+U_{x}+A U+B G=0, \\
G_{t}+2(G H)_{x}+G_{x x}+4 A\left(G_{x}-H_{x y}\right)+4 B\left(G_{y}-H_{y y}\right)+C\left(G-2 H_{y}\right)=0, \\
U_{y}-G_{x}=0,
\end{array}\right.
$$

where $A, B, C$ are constant parameters of the system.

Zhang et al. discussed the exact explicit solution and propagation in nonlinear model (2), and multi-solitoff solutions, multiple ring type localized solutions, multi-dromion solutions were obtained by the use of the truncated Painleve expansion and the variable separation approach. However, the variable separation form [(21) in [1]] these authors used to their equation [(20) in [1] ] is very particular, because it is very simplified. It is very natural to ask whether system (2) has other new solutions except those shown in Ref. [1]. The first attempt to answer this question would have been the work by Huang and Zhang [4], they used a new intermediate transformation and a variable-coefficient projective Riccati equation method to solve their so-called new (2+1)-dimensional simplified GBK system, which is a reduced form of (2) when $B=0$. They obtained new families of exact soliton-like solutions, then by imposing some conditions on these families of soliton-like solutions, some new exact solitary waves were given. The same question remained unsolved, since these authors solved a very specify system (2) with $B=0$.

On the other hand, in the study of the (2+1)-dimensional models, two kinds of "variable separating" procedure have been established. The first method called the "formal variable separation approach" (FVSA) [5], or equivalently the symmetry constraints or nonlinearization of the Lax-Pairs [6-8]. The independent variables of a reduced field in FVSA 
have not totally been separated though the reduced field satisfies some lower-dimensional equations. The second type of variable separation method has been established by Lou [9], he has proposed a multi-linear variable separation approach to search for exact solutions of the higher-dimensional especially (2+1)-dimensional NLPDEs. By solving the multi-linear form of these NLPDEs and introducing a prior ansatz, some special types of exact solutions can be obtained from two (1+1)-dimensional variable separated fields. This variable separation approach (VSA) is successfully applied to many (2+1)-dimensional integrable models such as the Davey Stewartson (DS) equation [10], the Nizhnik-Novikov-Veselov (NNV) equation [10], the dispersive long wave equation (DLWE) [11], the Broer-Kaup-Kupershmidt (BKK) system [12], the Burgers equation [13], the Maccari system [14], the general (N+M)components Ablowitz-Kaup-Newell-Segur (AKNS) system [15]. Because the formula of the field includes some arbitrary functions, abundant coherent structures such as, the solitoffs, dromions, lumps, ring-solitons, peakons, compactons, breathers and instantons are given. More recently, Tang and Lou have proposed a more general variable separation approach (GVSA) for several $(2+1)$-dimensional integrable models [13,16,17].

In this letter, we use the WTC truncation method and VSA (for $B \neq 0$ ) or GVSA (for $B=0$ ) to present another features of the (2+1)-dimensional GBK system (2). We are interested in the interactions of the coherent structures (solitons). As we know that soliton supplies good applied prospects in many fields of natural science such as plasmas, hydrodynamics, nonlinear optics, fiber optics, solid state physics, and the interactive property of soliton plays an important role in developing of many applications. Therefore, the study of the interactive property of soliton for integrable models is more significance. For this aim, will start as one of us did in two recent papers $[18,19]$, by the interaction properties of Jacobi elliptic waves, then the interaction of solitons are obtained as special case of the Jacobi elliptic waves.

The structure of the letter is elucidated as follows. In section 2, a summary of the properties of Jacobi elliptic function and that of the troncature of Painleve expansion are given. In Section 3, a general solution including two arbitrary functions is obtained for the $(2+1)$-dimensional simplified GBK system $(B=0)$ by means of WTC truncation method. By choosing appropriately these arbitrary functions, we study interaction properties of the periodic wave and the one of solitons. Section 4, is devoted to the study of the interaction of some other new localized structures to the $(2+1)$-dimensional GBK system (2). Some conclusion and discussion are given in the last section.

\section{Summary of the properties of Jacobi elliptic functions and that of the troncature of the Painlevé expansion}

The exact periodic wave solutions in terms of Jacobi elliptic functions for NLPDEs attract considerable interest [20-22] due to the elegant properties of the elliptic functions. The Jacobi elliptic functions $\operatorname{sn} \xi=\operatorname{sn}(\xi \mid m), c n \xi=c n(\xi \mid m)$ and $d n \xi=d n(\xi \mid m)$, where $m(0 \leq m \leq 1)$ is the modulus of the elliptic function, are double periodic and possess properties of trigonometric functions namely,

$s n^{2} \xi+c n^{2} \xi=1, d n^{2} \xi+m^{2} s^{2} \xi=1, \quad(s n \xi)^{\prime}=c n \xi d n \xi,(c n \xi)^{\prime}=-s n \xi d n \xi, \quad(d n \xi)^{\prime}=-m^{2} c n \xi s n \xi$. When $m \rightarrow 0$, the Jacobi elliptic functions degenerate into the trigonometric functions, i.e.

$s n \xi \rightarrow \sin \xi, c n \xi \rightarrow \cos \xi, d n \xi \rightarrow 1$. When $m \rightarrow 1$, the Jacobi elliptic functions degenerate into the hyperbolic functions, i.e. $\operatorname{sn} \xi \rightarrow \tanh \xi, c n \xi \rightarrow \operatorname{sech} \xi, d n \xi \rightarrow \operatorname{sech} \xi$. Detailed explanations about Jacobi elliptic functions can be found in Refs. [23,24]. The other nine Jacobi elliptic functions are all expressible in terms of $s n \xi, c n \xi$ and $d n \xi$ [23]. 
To conveniently solve the system (2), differentiating the first equation of this system with respect to y once and substituting the third equation of (2) into the resulting equation, we obtain the simplified form of(2) as follows

$$
\left\{\begin{array}{l}
\left(H_{t}-H_{x x}+2 H H_{x}\right)_{y}+G_{x x}+A G_{x}+B G_{y}=0 \\
G_{t}+2(G H)_{x}+G_{x x}+4 A\left(G_{x}-H_{x y}\right)+4 B\left(G-H_{y}\right)_{y}+C\left(G-2 H_{y}\right)=0 .
\end{array}\right.
$$

It is interesting to see that let $G=2 H_{y}$, Eq.(3) is decoupled into

$$
H_{t y}+2\left(H H_{x}\right)_{y}+H_{x x y}+2 A H_{x y}+2 B H_{y y}=0 .
$$

Then following the idea of WTC [25], we begin with the Painlevé expansion of Eq.(4) truncated at the constant term

$$
H=\varphi^{-1} H_{0}+H_{1},
$$

where $\varphi \equiv \varphi(x, y, t)$ is the singular manifold variable, $H_{0}$ and $H_{1}$ the functions of arguments $\mathrm{x}, \mathrm{y}$ and $\mathrm{t}$. The substitution of (5) into (4) leads to five equations depending of the different powers of $\varphi$. At the powers $\varphi^{-4}$ and $\varphi^{0}$, the following results

$$
\begin{gathered}
H_{0}=\varphi_{x}, \\
\left(H_{1 t}+2 H_{1} H_{1 x}+H_{1 x x}+2 A H_{1 x}+2 B H_{1 y}\right)_{y}=0,
\end{gathered}
$$

are obtained respectively. By choosing $H_{1} \equiv H_{1}(x, t)$, Eq. (7) is satisfied automatically. After that, using (6) into the three remaining equations, the following results are obtained

$$
\left\{\begin{array}{cl}
\varphi^{-3}: & \varphi_{x} \varphi_{y}\left(\varphi_{t}+2 A \varphi_{x}+2 H_{1} \varphi_{x}+\varphi_{x x}+2 B \varphi_{y}\right)=0, \\
\varphi^{-2}: & \varphi_{x}\left(\varphi_{t}+2 A \varphi_{x}+2 H_{1} \varphi_{x}+\varphi_{x x}+2 B \varphi_{y}\right)_{y}+\varphi_{y}\left(\varphi_{t}+2 A \varphi_{x}+2 H_{1} \varphi_{x}+\varphi_{x x}+2 B \varphi_{y}\right)_{x}+ \\
& \varphi_{x y}\left(\varphi_{t}+2 A \varphi_{x}+2 H_{1} \varphi_{x}+\varphi_{x x}+2 B \varphi_{y}\right)=0, \\
\varphi^{-1}: & \left(\varphi_{t}+2 A \varphi_{x}+2 H_{1} \varphi_{x}+\varphi_{x x}+2 B \varphi_{y}\right)_{x y}=0 .
\end{array}\right.
$$

Analysing above system, we find that all equations in this system are satisfied automacally under the conditions of

$$
\varphi_{t}+2 A \varphi_{x}+2 H_{1} \varphi_{x}+\varphi_{x x}+2 B \varphi_{y}=0 .
$$

To carry out the resolution of Eq.(9), we will consider two situations: The case $B=0$, the system is reduced to the so-called (2+1)-dimensional simplified GBK system and when $B \neq 0$ the system is the so-called (2+1)-dimensional GBK system. The readers can see in what follows that in these two cases the behaviour of the solutions is quite different in the system.

\section{General solutions of the (2+1)-dimensional simplified GBK system}

When $B=0$, Eq.(9) is reduced to

$$
\varphi_{t}+2 A \varphi_{x}+2 H_{1} \varphi_{x}+\varphi_{x x}=0
$$


About the linear equation (10) of the system (2) in which condition $B=0$, we can construct many types of special solutions. Because $H_{1}(x, t)$ is undetermined function of variable $\mathrm{x}$ and $\mathrm{t}$, we can select after a careful analysis, an appropriate variable separation hypothesis for the function $\varphi$ as follows

$$
\varphi(x, y, t)=f(x, t) g(y)+h(y)
$$

where $f, g$ and $h$ are arbitrary functions of indicated variables. If we take the function $H_{1}$ as

$$
H_{1}=-\frac{f_{t}+2 A f_{x}+f_{x x}}{2 f_{x}}
$$

So the general solution of the (2+1)-dimensional simplified GBK system is found as follows

$$
\begin{aligned}
H & =\frac{f_{x} g}{f g+h}-\frac{f_{t}+2 A f_{x}+f_{x x}}{2 f_{x}}, \\
G & =2 H_{y}=2 \frac{f_{x}\left(h g_{y}-g h_{y}\right)}{(f g+h)^{2}}, \\
U & =2 H_{x}+K(x, t) \\
& =2 \frac{g\left\{f_{x x}(f g+h)-f_{x}^{2} g\right\}}{(f g+h)^{2}}-\frac{f_{x}\left(f_{x t}+f_{x x x}\right)-f_{x x}\left(f_{t}+f_{x x}\right)}{f_{x}^{2}}+K(x, t),
\end{aligned}
$$

where $K(x, t)$ is an arbitrary function.

Because of the arbitrariness of the functions $f, g$ and $h$ appearing in Eq.(13), the potential $G$ possesses quite rich structures. In the following, we may obtain a diversity of periodic wave solutions in terms of the Jacobi elliptic functions by selecting the arbitrary functions appropriately. It is worth noticing that the Jacobi transformation

$d n(\xi \mid m)=c n\left(\sqrt{m} \xi \mid m^{-1}\right)$ [23] implies that any solution found by the $d n$ - function may be transformed into an equivalent one that can be obtained by $c n$ - function. Moreover, since other Jacobi elliptic functions have singularities, we consider only the periodic wave solutions in terms of $s n$ - and $c n$ - functions. We will focus in our study only on solutions representing the interaction between the Jacobi elliptic waves with different speeds, the other solutions of the potential $\mathrm{g}$ can be seen in [18].

\subsection{Exact Periodic Wave Solutions}

Case 1. $f=c n\left(k_{1} x-\omega_{1} t \mid M_{1}\right)+c n\left(k_{2} x-\omega_{2} t \mid M_{2}\right)=c n\left(\xi_{1}\right)+c n\left(\xi_{2}\right), g=c n\left(l_{1} y \mid m_{1}\right)=c n\left(\eta_{1}\right)$,

$$
h=D+c n\left(l_{2} y \mid m_{2}\right)=D+c n\left(\eta_{2}\right) .
$$

The new type of periodic wave solution, representing the interaction between the Jacobi elliptic waves with different speeds reads

$G=\frac{\left(-\sum_{i=1}^{2} k_{i} \operatorname{sn}\left(\xi_{i}\right) d n\left(\xi_{i}\right)\right)\left\{-l_{1} \operatorname{sn}\left(\eta_{1}\right) d n\left(\eta_{1}\right)\left(D+c n\left(\eta_{2}\right)\right)+l_{2} c n\left(\eta_{1}\right) \operatorname{sn}\left(\eta_{2}\right) d n\left(\eta_{2}\right)\right\}}{\left\{\left(\operatorname{cn}\left(\xi_{1}\right)+\operatorname{cn}\left(\xi_{2}\right)\right) \operatorname{cn}\left(\eta_{1}\right)+D+c n\left(\eta_{2}\right)\right\}^{2}}$, 
where $k_{i}, l_{i}, \omega_{i}(i=1,2)$ and $D$ are arbitrary constants, and $m_{i}, M_{i}(i=1,2)$ the modulus of the elliptic function, and the constant $D$ guarantees that the solution has no singularity (because $s n-$ and $c n$-functions have zeros).

Case 2. $f=\operatorname{cn}\left(k_{1} x-\omega_{1} t \mid M_{1}\right)+c n\left(k_{2} x-\omega_{2} t \mid M_{2}\right)=\operatorname{cn}\left(\xi_{1}\right)+c n\left(\xi_{2}\right), g=\operatorname{sn}\left(l_{1} y \mid m_{1}\right)=\operatorname{sn}\left(\eta_{1}\right)$,

$$
h=D+\operatorname{sn}\left(l_{2} y \mid m_{2}\right)=D+\operatorname{sn}\left(\eta_{2}\right) .
$$

From the second equation of (13), we have

$$
G=\frac{\left(-\sum_{i=1}^{2} k_{i} \operatorname{sn}\left(\xi_{i}\right) d n\left(\xi_{i}\right)\right)\left\{l_{1} c n\left(\eta_{1}\right) d n\left(\eta_{1}\right)\left(D+\operatorname{sn}\left(\eta_{2}\right)\right)-l_{2} \operatorname{sn}\left(\eta_{1}\right) c n\left(\eta_{2}\right) d n\left(\eta_{2}\right)\right\}}{\left\{\left(\operatorname{cn}\left(\xi_{1}\right)+c n\left(\xi_{2}\right)\right) \operatorname{sn}\left(\eta_{1}\right)+D+\operatorname{sn}\left(\eta_{2}\right)\right\}^{2}} .
$$

Case 3. $f=c n\left(k_{1} x-\omega_{1} t \mid M_{1}\right)+c n\left(k_{2} x-\omega_{2} t \mid M_{2}\right)=c n\left(\xi_{1}\right)+c n\left(\xi_{2}\right), g=c n\left(l_{1} y \mid m_{1}\right)=c n\left(\eta_{1}\right)$,

$$
h=D+\operatorname{sn}\left(l_{2} y \mid m_{2}\right)=D+\operatorname{sn}\left(\eta_{2}\right) .
$$

The other new periodic wave solution of the system (2) with $B=0$ is given by

$$
G=\frac{\left(-\sum_{i=1}^{2} k_{i} \operatorname{sn}\left(\xi_{i}\right) d n\left(\xi_{i}\right)\right)\left\{-l_{1} \operatorname{sn}\left(\eta_{1}\right) d n\left(\eta_{1}\right)\left(D+\operatorname{sn}\left(\eta_{2}\right)\right)-l_{2} \operatorname{cn}\left(\eta_{1}\right) \operatorname{cn}\left(\eta_{2}\right) d n\left(\eta_{2}\right)\right\}}{\left\{\left(\operatorname{cn}\left(\xi_{1}\right)+\operatorname{cn}\left(\xi_{2}\right)\right) \operatorname{cn}\left(\eta_{1}\right)+D+\operatorname{cn}\left(\eta_{2}\right)\right\}^{2}} .
$$

Case 4. $f=\operatorname{cn}\left(k_{1} x-\omega_{1} t \mid M_{1}\right)+c n\left(k_{2} x-\omega_{2} t \mid M_{2}\right)=\operatorname{cn}\left(\xi_{1}\right)+c n\left(\xi_{2}\right), g=\operatorname{sn}\left(l_{1} y \mid m_{1}\right)=\operatorname{sn}\left(\eta_{1}\right)$,

$$
h=D+c n\left(l_{2} y \mid m_{2}\right)=D+c n\left(\eta_{2}\right) .
$$

From the second equation of (13), we have

$$
G=\frac{\left(-\sum_{i=1}^{2} k_{i} \operatorname{sn}\left(\xi_{i}\right) d n\left(\xi_{i}\right)\right)\left\{l_{1} c n\left(\eta_{1}\right) d n\left(\eta_{1}\right)\left(D+c n\left(\eta_{2}\right)\right)+l_{2} \operatorname{sn}\left(\eta_{1}\right) \operatorname{sn}\left(\eta_{2}\right) d n\left(\eta_{2}\right)\right\}}{\left\{\left(\operatorname{cn}\left(\xi_{1}\right)+c n\left(\xi_{2}\right)\right) \operatorname{sn}\left(\eta_{1}\right)+D+c n\left(\eta_{2}\right)\right\}^{2}} .
$$

Case 5. $f=\operatorname{sn}\left(k_{1} x-\omega_{1} t \mid M_{1}\right)+\operatorname{sn}\left(k_{2} x-\omega_{2} t \mid M_{2}\right)=\operatorname{sn}\left(\xi_{1}\right)+\operatorname{sn}\left(\xi_{2}\right), g=\operatorname{sn}\left(l_{1} y \mid m_{1}\right)=\operatorname{sn}\left(\eta_{1}\right)$,

$$
h=D+\operatorname{sn}\left(l_{2} y \mid m_{2}\right)=D+\operatorname{sn}\left(\eta_{2}\right)
$$

The new type of periodic wave solution, representing the interaction between the Jacobi elliptic waves with different speeds reads

$$
G=\frac{\left(\sum_{i=1}^{2} k_{i} \operatorname{cn}\left(\xi_{i}\right) d n\left(\xi_{i}\right)\right)\left\{l_{1} \operatorname{cn}\left(\eta_{1}\right) \operatorname{dn}\left(\eta_{1}\right)\left(D+\operatorname{sn}\left(\eta_{2}\right)\right)-l_{2} \operatorname{sn}\left(\eta_{1}\right) c n\left(\eta_{2}\right) d n\left(\eta_{2}\right)\right\}}{\left\{\left(\operatorname{sn}\left(\xi_{1}\right)+\operatorname{sn}\left(\xi_{2}\right)\right) \operatorname{sn}\left(\eta_{1}\right)+D+\operatorname{sn}\left(\eta_{2}\right)\right\}^{2}},
$$

The other new periodic wave solution of the system (2) with $B=0$ is

Case 6. $f=\operatorname{sn}\left(k_{1} x-\omega_{1} t \mid M_{1}\right)+\operatorname{sn}\left(k_{2} x-\omega_{2} t \mid M_{2}\right)=\operatorname{sn}\left(\xi_{1}\right)+\operatorname{sn}\left(\xi_{2}\right), g=c n\left(l_{1} y \mid m_{1}\right)=\operatorname{cn}\left(\eta_{1}\right)$, 


$$
h=D+c n\left(l_{2} y \mid m_{2}\right)=D+c n\left(\eta_{2}\right) .
$$

From the second equation of (13), we have

$$
G=\frac{\left(\sum_{i=1}^{2} k_{i} \operatorname{cn}\left(\xi_{i}\right) d n\left(\xi_{i}\right)\right)\left\{-l_{1} \operatorname{sn}\left(\eta_{1}\right) d n\left(\eta_{1}\right)\left(D+\operatorname{cn}\left(\eta_{2}\right)\right)+l_{2} \operatorname{cn}\left(\eta_{1}\right) \operatorname{sn}\left(\eta_{2}\right) d n\left(\eta_{2}\right)\right\}}{\left\{\left(\operatorname{sn}\left(\xi_{1}\right)+\operatorname{sn}\left(\xi_{2}\right)\right) \operatorname{cn}\left(\eta_{1}\right)+D+\operatorname{cn}\left(\eta_{2}\right)\right\}^{2}} .
$$

Case 7. $f=\operatorname{sn}\left(k_{1} x-\omega_{1} t \mid M_{1}\right)+\operatorname{sn}\left(k_{2} x-\omega_{2} t \mid M_{2}\right)=\operatorname{sn}\left(\xi_{1}\right)+\operatorname{sn}\left(\xi_{2}\right), g=c n\left(l_{1} y \mid m_{1}\right)=\operatorname{cn}\left(\eta_{1}\right)$,

$$
h=D+\operatorname{sn}\left(l_{2} y \mid m_{2}\right)=D+\operatorname{sn}\left(\eta_{2}\right) .
$$

We have the periodic wave solution of the system (2) with $B=0$

$$
G=\frac{\left(\sum_{i=1}^{2} k_{i} \operatorname{cn}\left(\xi_{i}\right) d n\left(\xi_{i}\right)\right)\left\{-l_{1} \operatorname{sn}\left(\eta_{1}\right) d n\left(\eta_{1}\right)\left(D+\operatorname{sn}\left(\eta_{2}\right)\right)-l_{2} c n\left(\eta_{1}\right) c n\left(\eta_{2}\right) d n\left(\eta_{2}\right)\right\}}{\left\{\left(\operatorname{sn}\left(\xi_{1}\right)+\operatorname{sn}\left(\xi_{2}\right)\right) \operatorname{cn}\left(\eta_{1}\right)+D+\operatorname{sn}\left(\eta_{2}\right)\right\}^{2}} .
$$

Case 8. $f=\operatorname{sn}\left(k_{1} x-\omega_{1} t \mid M_{1}\right)+\operatorname{sn}\left(k_{2} x-\omega_{2} t \mid M_{2}\right)=\operatorname{sn}\left(\xi_{1}\right)+\operatorname{sn}\left(\xi_{2}\right), g=\operatorname{sn}\left(l_{1} y \mid m_{1}\right)=\operatorname{sn}\left(\eta_{1}\right)$,

$$
h=D+c n\left(l_{2} y \mid m_{2}\right)=D+c n\left(\eta_{2}\right) .
$$

The periodic wave solution of the system (2) with $B=0$ is

$$
G=\frac{\left(\sum_{i=1}^{2} k_{i} \operatorname{cn}\left(\xi_{i}\right) d n\left(\xi_{i}\right)\right)\left\{l_{1} c n\left(\eta_{1}\right) d n\left(\eta_{1}\right)\left(D+\operatorname{cn}\left(\eta_{2}\right)\right)+l_{2} \operatorname{sn}\left(\eta_{1}\right) \operatorname{sn}\left(\eta_{2}\right) d n\left(\eta_{2}\right)\right\}}{\left\{\left(\operatorname{sn}\left(\xi_{1}\right)+\operatorname{sn}\left(\xi_{2}\right)\right) \operatorname{sn}\left(\eta_{1}\right)+D+\operatorname{cn}\left(\eta_{2}\right)\right\}^{2}} .
$$

After plotting the eight solutions (14)-(21), it appears that the evolution behaviour of their interaction is non-elastic. Let us select as example one of them, the figure (1). This figure shows the detail on the interaction property of the Jacobi elliptic solutions (15), when selecting the parameter values $k_{1}=l_{1}=1, k_{2}=l_{2}=2, \omega_{1}=\omega_{2}=1, D=4$, $M_{1}=0.2, M_{2}=0.3, m_{1}=0.25, m_{2}=0.35$, and the corresponding time is indicated on this figure.

\subsection{Solitary Wave Solutions}

In this part, we study limit cases of Jacobi elliptic wave solutions obtained above. Due to the limit of the space, we discuss only cases which new and different interactions of waves. As $M_{1}, M_{2}, m_{1}$ and $m_{2}$ approach 1, it follows from (14), (15), (19) and (21) that

$$
G=\frac{\left(-\sum_{i=1}^{2} k_{i} \operatorname{sech}\left(k_{i} x-\omega_{i} t\right) \tanh \left(k_{i} x-\omega_{i} t\right)\right)\left\{-l_{1} \tanh \left(l_{1} y\right) \operatorname{sech}\left(l_{1} y\right)\left(D+\operatorname{sech}\left(l_{2} y\right)\right)+l_{2} \operatorname{sech}\left(l_{1} y\right) \tanh \left(l_{2} y\right) \operatorname{sech}\left(l_{2} y\right)\right\}}{\left\{\left(\operatorname{sech}\left(k_{1} x-\omega_{1} t\right)+\operatorname{sech}\left(k_{2} x-\omega_{2} t\right)\right) \operatorname{sech}\left(l_{1} y\right)+D+\operatorname{sech}\left(l_{2} y\right)\right\}^{2}}
$$

$$
G=\frac{\left(-\sum_{i=1}^{2} k_{i} \operatorname{sech}\left(k_{i} x-\omega_{i} t\right) \tanh \left(k_{i} x-\omega_{i} t\right)\right)\left\{l_{1} \operatorname{sech}^{2}\left(l_{1} y\right)\left(D+\tanh \left(l_{2} y\right)\right)-l_{2} \tanh \left(l_{1} y\right) \operatorname{sech}^{2}\left(l_{2} y\right)\right\}}{\left\{\left(\operatorname{sech}\left(k_{1} x-\omega_{1} t\right)+\operatorname{sech}\left(k_{2} x-\omega_{2} t\right)\right) \tanh \left(l_{1} y\right)+D+\tanh \left(l_{2} y\right)\right\}^{2}},
$$


$G=\frac{\left(\sum_{i=1}^{2} k_{i} \operatorname{sech}^{2}\left(k_{i} x-\omega_{i} t\right)\right)\left\{-l_{1} \tanh \left(l_{1} y\right) \operatorname{sech}\left(l_{1} y\right)\left(D+\operatorname{sech}\left(l_{2} y\right)\right)+l_{2} \operatorname{sech}\left(l_{1} y\right) \tanh \left(l_{2} y\right) \operatorname{sech}\left(l_{2} y\right)\right\}}{\left\{\left(\tanh \left(k_{1} x-\omega_{1} t\right)+\tanh \left(k_{2} x-\omega_{2} t\right)\right) \operatorname{sech}\left(l_{1} y\right)+D+\operatorname{sech}\left(l_{2} y\right)\right\}^{2}}$,

$G=\frac{\left(\sum_{i=1}^{2} k_{i} \operatorname{sech}^{2}\left(k_{i} x-\omega_{i} t\right)\right)\left\{l_{1} \operatorname{sech}^{2}\left(l_{1} y\right)\left(D+\operatorname{sech}\left(l_{2} y\right)\right)+l_{2} \tanh \left(l_{1} y\right) \tanh \left(l_{2} y\right) \operatorname{sech}\left(l_{2} y\right)\right\}}{\left\{\left(\tanh \left(k_{1} x-\omega_{1} t\right)+\tanh \left(k_{2} x-\omega_{2} t\right)\right) \tanh \left(l_{1} y\right)+D+\operatorname{sech}\left(l_{2} y\right)\right\}^{2}}$,

Are the new solutions respectively.

As $M_{1}, M_{2} \rightarrow 1$ and $m_{1}, m_{2} \rightarrow 0$, we obtain from Eqs. (14) and (19) two new solutions given by

$$
\begin{gathered}
G=\frac{\left(-\sum_{i=1}^{2} k_{i} \operatorname{sech}\left(k_{i} x-\omega_{i} t\right) \tanh \left(k_{i} x-\omega_{i} t\right)\right)\left\{-l_{1} \sin \left(l_{1} y\right)\left(D+\cos \left(l_{2} y\right)\right)+l_{2} \cos \left(l_{1} y\right) \sin \left(l_{2} y\right)\right\}}{\left\{\left(\operatorname{sech}\left(k_{1} x-\omega_{1} t\right)+\operatorname{sech}\left(k_{2} x-\omega_{2} t\right)\right) \cos \left(l_{1} y\right)+D+\cos \left(l_{2} y\right)\right\}^{2}}, \\
G=\frac{\left(\sum_{i=1}^{2} k_{i} \operatorname{sech}^{2}\left(k_{i} x-\omega_{i} t\right)\right)\left\{-l_{1} \sin \left(l_{1} y\right)\left(D+\cos \left(l_{2} y\right)\right)+l_{2} \cos \left(l_{1} y\right) \sin \left(l_{2} y\right)\right\}}{\left\{\left(\tanh \left(k_{1} x-\omega_{1} t\right)+\tanh \left(k_{2} x-\omega_{2} t\right)\right) \cos \left(l_{1} y\right)+D+\cos \left(l_{2} y\right)\right\}^{2}},
\end{gathered}
$$

Respectively.

As $M_{1}, M_{2} \rightarrow 0$ and $m_{1}, m_{2} \rightarrow 1$, we obtain from Eqs. (15) a new solution given by

$G=\frac{\left(-\sum_{i=1}^{2} k_{i} \sin \left(k_{i} x-\omega_{i} t\right)\right)\left\{l_{1} \operatorname{sech}^{2}\left(l_{1} y\right)\left(D+\tanh \left(l_{2} y\right)\right)-l_{2} \tanh \left(l_{1} y\right) \operatorname{sech}^{2}\left(l_{2} y\right)\right\}}{\left\{\left(\cos \left(k_{1} x-\omega_{1} t\right)+\cos \left(k_{2} x-\omega_{2} t\right)\right) \tanh \left(l_{1} y\right)+D+\tanh \left(l_{2} y\right)\right\}^{2}}$.

As $M_{1}, M_{2}, m_{2} \rightarrow 1$ and $m_{1} \rightarrow 0$, we obtain from Eq. (19), another new solution given by the following relation

$G=\frac{\left(\sum_{i=1}^{2} k_{i} \operatorname{sech}^{2}\left(k_{i} x-\omega_{i} t\right)\right)\left\{-l_{1} \sin \left(l_{1} y\right)\left(D+\operatorname{sech}\left(l_{2} y\right)\right)+l_{2} \cos \left(l_{1} y\right) \tanh \left(l_{2} y\right) \operatorname{sech}\left(l_{2} y\right)\right\}}{\left\{\left(\tanh \left(k_{1} x-\omega_{1} t\right)+\tanh \left(k_{2} x-\omega_{2} t\right)\right) \cos \left(l_{1} y\right)+D+\operatorname{sech}\left(l_{2} y\right)\right\}^{2}}$.

It is important to say that mathematically, there are many solutions corresponding to the degeneracy of the eight solutions (14)-(21), but we have written down explicitly only those which plots show new behaviour for the model. The evolution behaviour of the interactions shown in figures (2), (4) (6) and (9) is non-elastic, while that of figures (3), (5) is elastic. 
$t=-5$
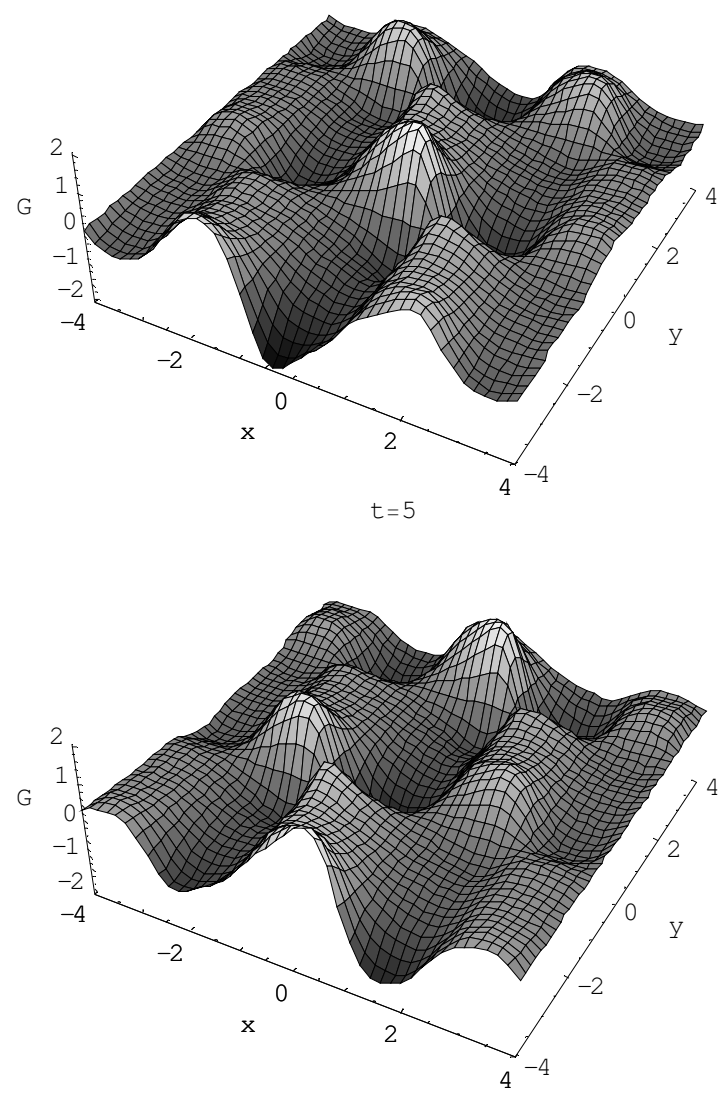

Fig.1. The evolution of periodic wave solution (15).
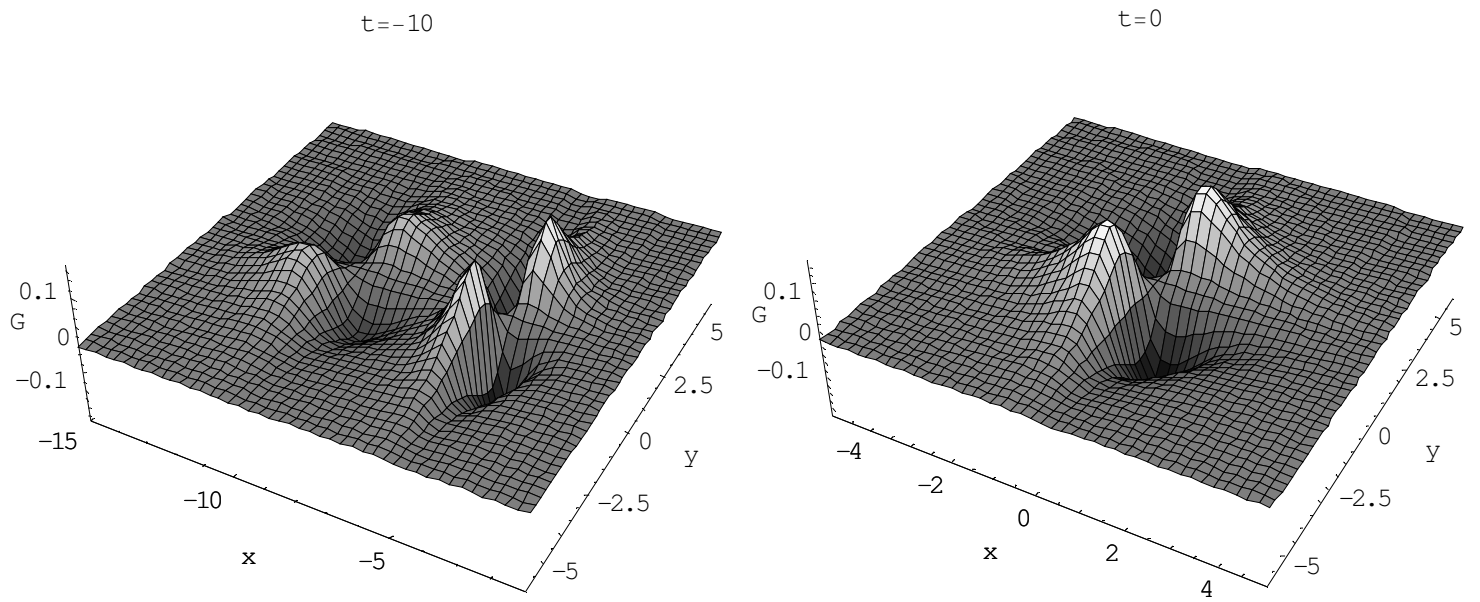


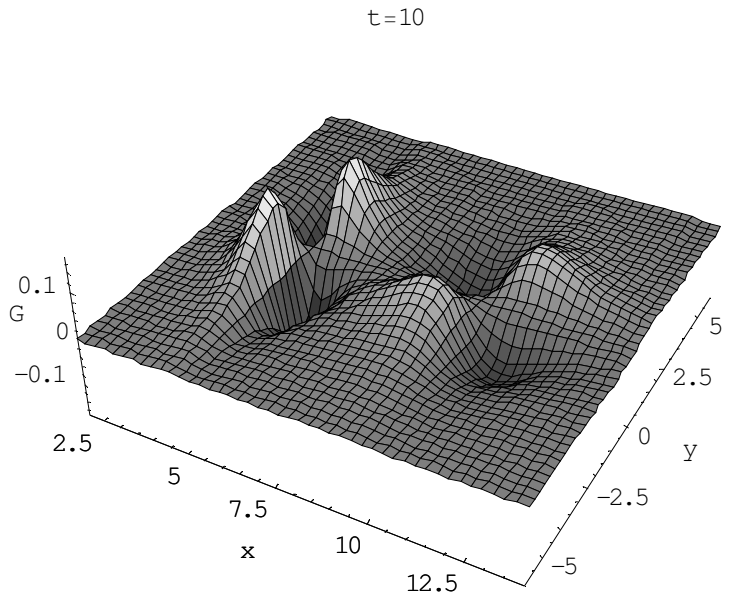

Fig.2. The evolution of two solitonic excitation for the potential field G given by (22).
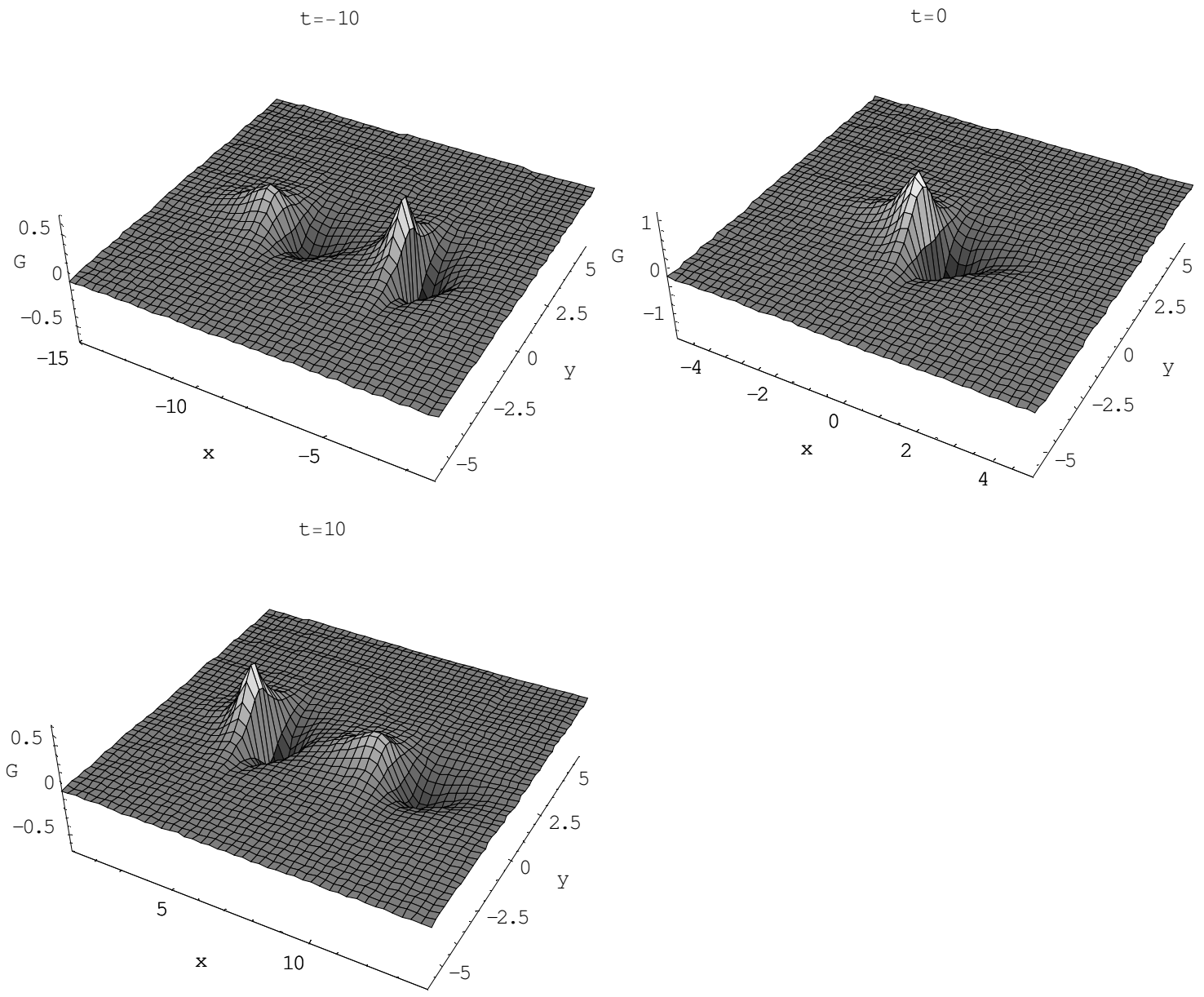

Fig.3. The evolution of two solitonic excitation for the potential field G given by (23). 

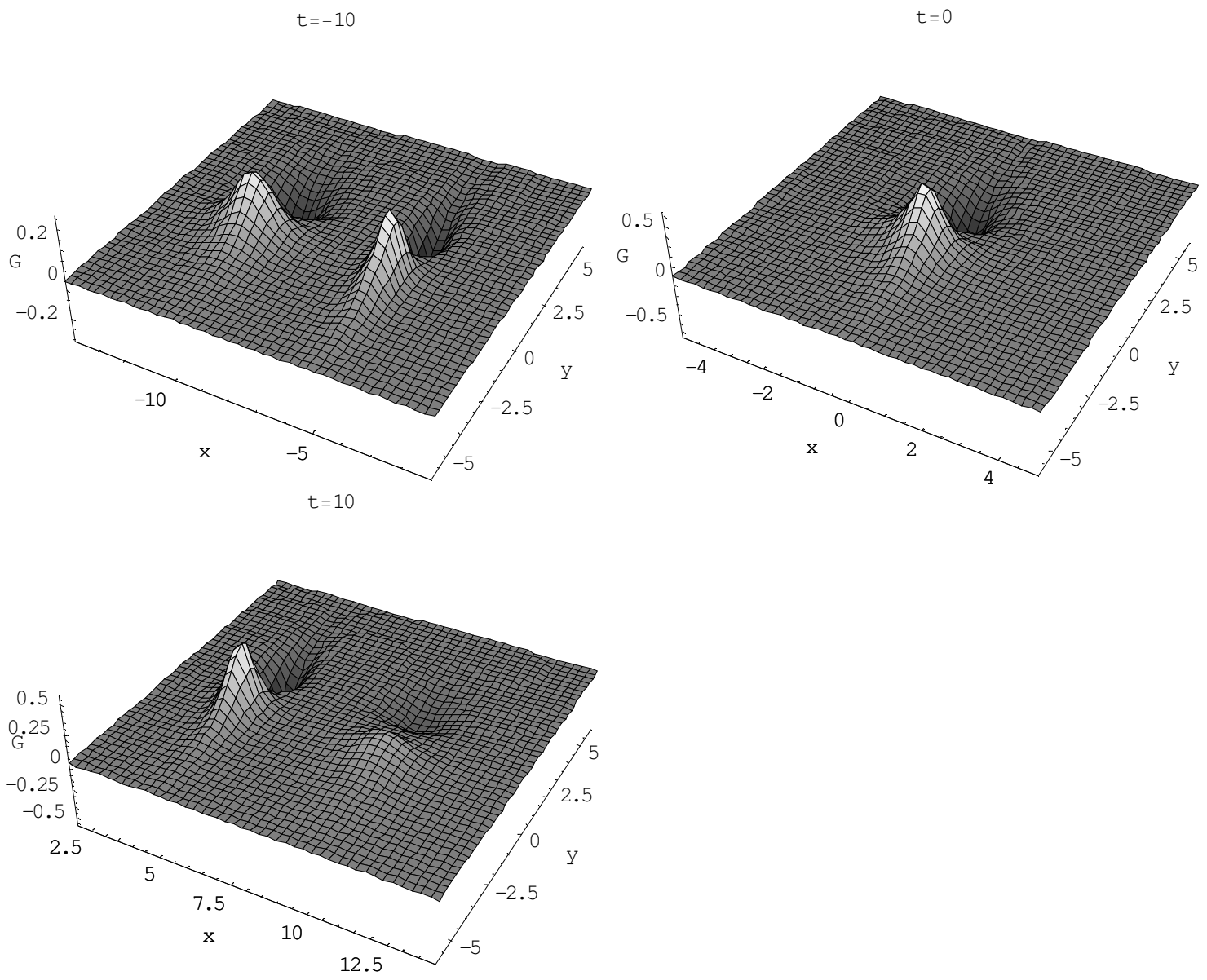

Fig.4. The evolution of two solitonic excitation for the potential field G given by (24).

$t=-10$

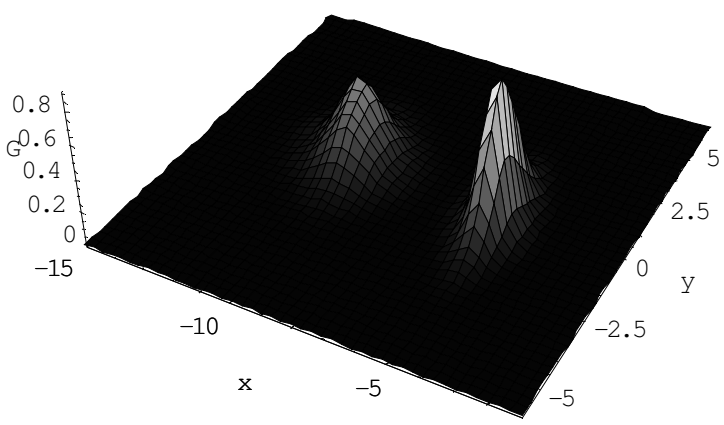

$t=0$

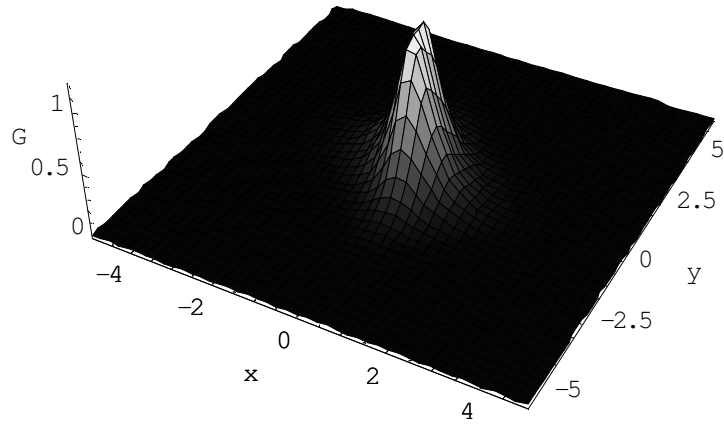




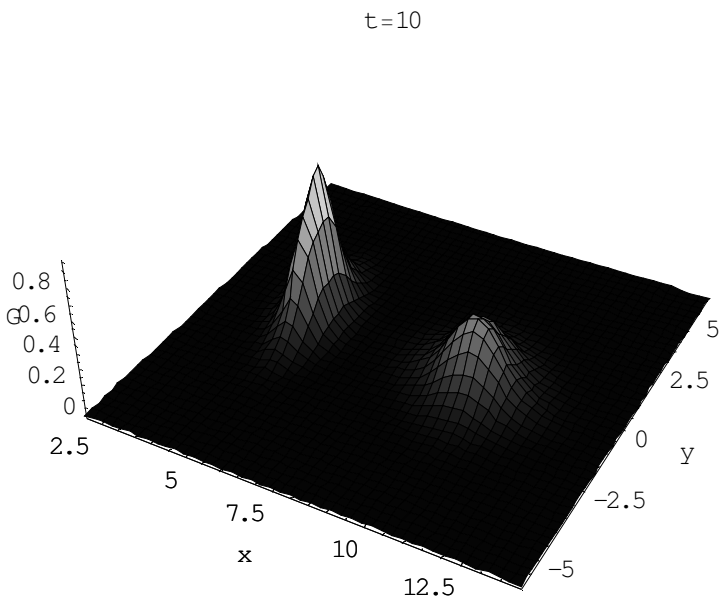

Fig.5. The evolution of two dromions for the potential field $\mathrm{G}$ given by (25).

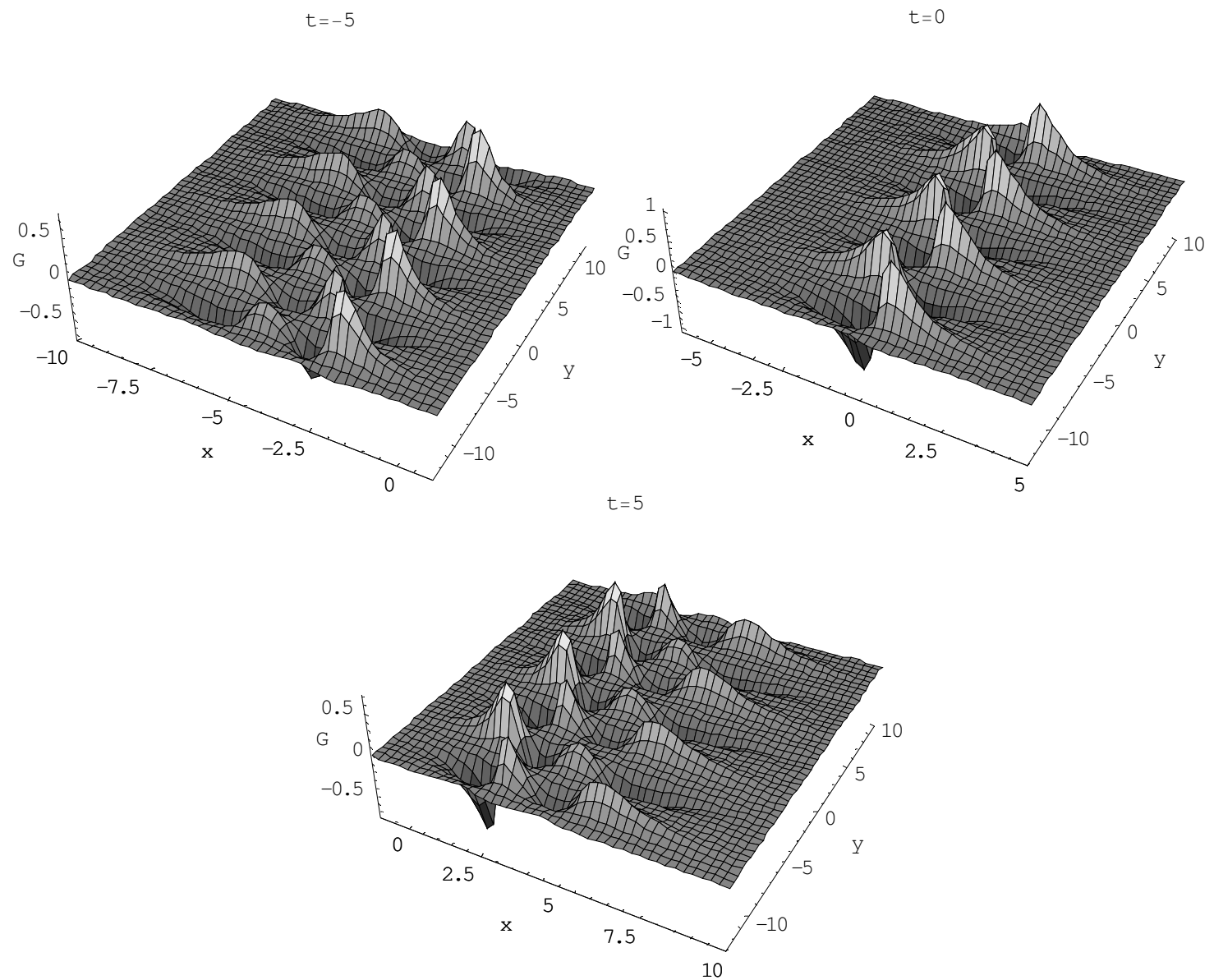

Fig.6. The evolution of the group of y periodic-solitons (26). 


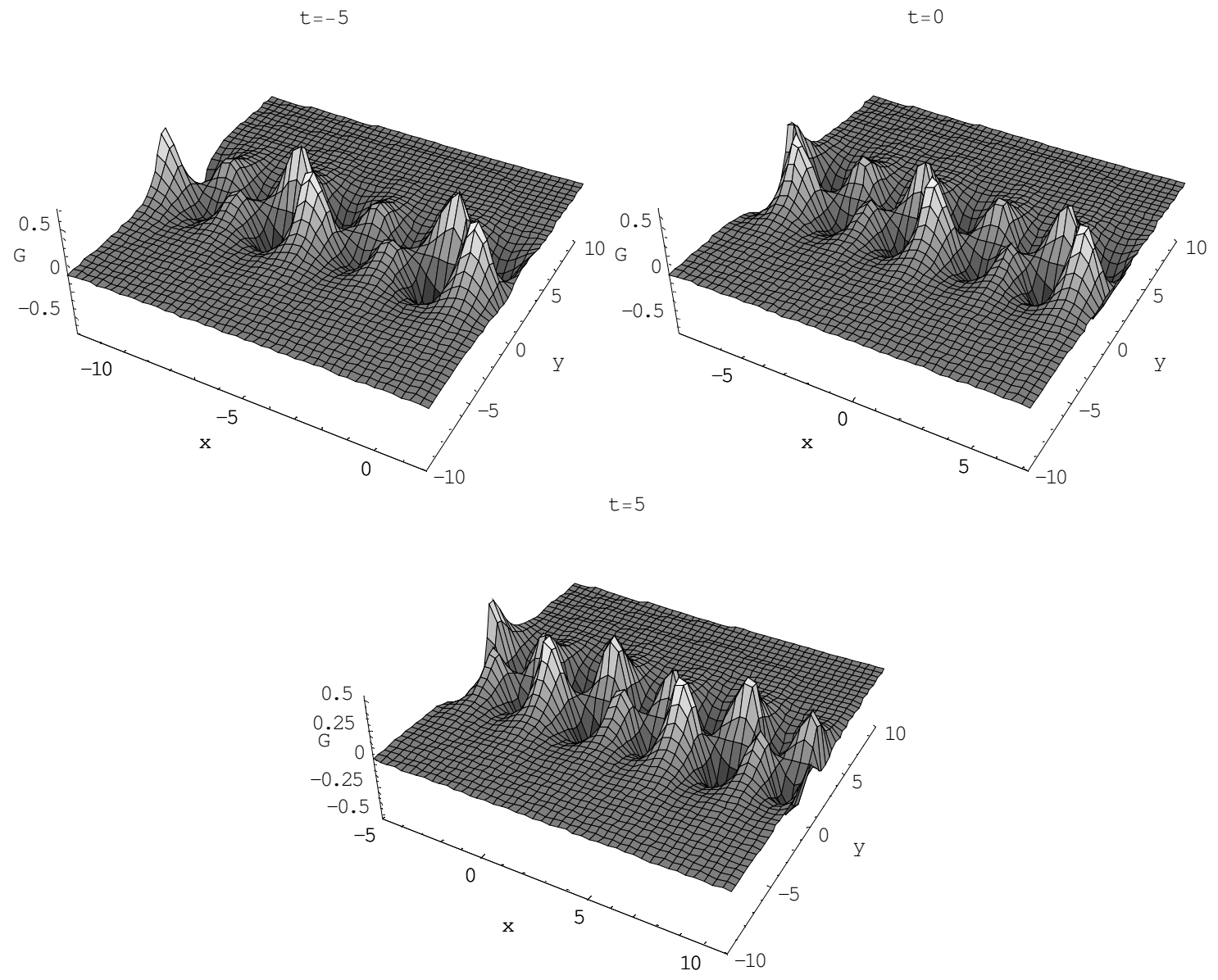

Fig.7. The evolution of $x$-periodic solitons (27).

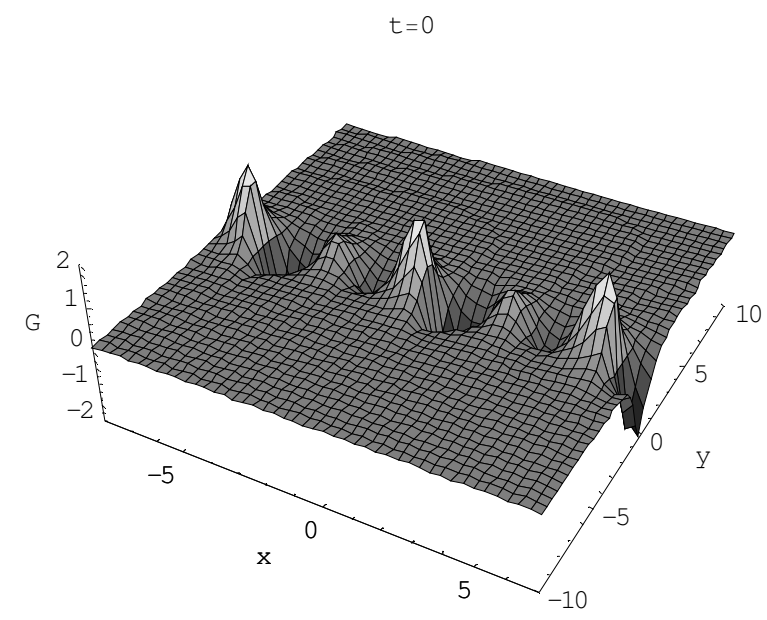

Fig.8. A typical spatial structure of y-periodic solitons (28). 




Fig.9. The evolution of another group of y periodic-solitons (29).

\section{General solutions of the (2+1)-dimensional GBK system}

When $B \neq 0$, after obtaining the linear equation (9) of the original system (2), we select an appropriate variable separation hypothesis for the function $\varphi$. We will follow in our choice, a modifying Hirota's multi-soliton form used for many integrable models [9],

$\varphi(x, y, t)=a_{0}+a_{1} f(x, t)+a_{2} g(y, t)+a_{3} f(x, t) g(y, t)$,

where $f$ and $g$ are arbitrary functions of indicated variables, $a_{0}, a_{1}, a_{2}$ and $a_{3}$ are arbitrary constants. Inserting the ansatz (30) into Eq.(9) we finally obtain the following two simple variable separated equations

$$
\begin{gathered}
f_{t}+2 A f_{x}+2 H_{1} f_{x}+f_{x x}-K_{1}\left(a_{2}+a_{3} f\right)=0, \\
g_{t}+2 B g_{y}+K_{1}\left(a_{1}+a_{3} g\right)=0,
\end{gathered}
$$

where $K_{1}$ is an arbitrary constant.

It is easy to obtain general solutions of Eqs.(31) and (32). Since $H_{1}$ is arbitrary seed solution, we can view $f$ as an arbitrary function of $\mathrm{x}$ and $\mathrm{t}$ then the seed solution $H_{1}$ can be fixed by Eq.(31), which reads 


$$
\mathrm{H}_{1}=\frac{K_{1}\left(a_{2}+a_{3} f\right)-\left(f_{t}+2 A f_{x}+f_{x x}\right)}{2 f_{x}} .
$$

As to Eq.(32), its general solution has the form

$$
g=-\frac{a_{1}}{a_{3}}+\frac{1}{a_{3}} g_{0}(y-2 B t) \exp \left(-\frac{a_{3} K_{1}}{2 B} y\right),
$$

where $g_{0}(y-2 B t)$ is an arbitrary function of $y-2 B t$.

Finally, we obtain a general solution of the (2+1)-dimensional GBK equation

$$
\begin{aligned}
H & =\frac{\left(a_{1}+a_{3} g\right) f_{x}}{a_{0}+a_{1} f+a_{2} g+a_{3} f g}+\frac{K_{1}\left(a_{2}+a_{3} f\right)-\left(f_{t}+2 A f_{x}+f_{x x}\right)}{2 f_{x}}, \\
G & =2 H_{y}=2 \frac{f_{x} g_{y}\left(a_{0} a_{3}-a_{1} a_{2}\right)}{\left(a_{0}+a_{1} f+a_{2} g+a_{3} f g\right)^{2}}, \\
U & =2 H_{x}+K(x, t) \\
& =2 \frac{\left(a_{1}+a_{3} g\right)\left\{f_{x x}\left(a_{0}+a_{1} f+a_{2} g+a_{3} f g\right)-f_{x}^{2}\left(a_{1}+a_{3} g\right)\right\}}{\left(a_{0}+a_{1} f+a_{2} g+a_{3} f g\right)^{2}}+\frac{f_{x}\left\{K_{1} a_{3} f_{x}-\left(f_{x t}+f_{x x}\right)\right\}+f_{x x}\left\{f_{t}+f_{x x}-K_{1}\left(a_{2}+a_{3} f\right)\right\}}{f_{x}^{2}}+K(x, t),
\end{aligned}
$$

with three arbitrary functions $f(x, t), g(y, t)$ and $K(x, t)$.

\subsection{Novel Solutions of (2+1)-Dimensional GBK system}

Here, we investigate the interaction behaviour of the other new structures for the potential field $G$. If we select $f$ and $g$ as

$$
\begin{aligned}
& f=-\frac{a_{1}}{a_{3}}+\ln \left\{b_{0}+\sum_{i=1}^{N} b_{i} \exp \left(k_{i} x-\omega_{i} t+x_{0 i}\right)\right\}_{x x}, \\
& g=-\frac{a_{1}}{a_{3}}+\frac{1}{a_{3}} \exp \left(-\frac{a_{3} K_{1}}{2 B} y\right) \ln \left\{c_{0}+\sum_{j=1}^{M} c_{j} \exp \left(L_{j}(y-2 B t)+y_{0 j}\right)\right\}_{y y},
\end{aligned}
$$

where $k_{i}, \omega_{i}, L_{j}, b_{i}, c_{j}, x_{0 i}, y_{0 j}, a_{1}, a_{3}, c_{0}, b_{0}, K_{1}$ and $B$ are arbitrary constants and $M, N$ are arbitrary integers, when choosing

$N=3, M=1, b_{0}=c_{0}=c_{1}=2 b_{1}=2 b_{2}=2 L_{1}=b_{3}=1, \quad k_{1}=-1 / 8, k_{2}=2, k_{3}=5, \omega_{2}=-\omega_{1}=3$,

$\omega_{3}=2, x_{01}=x_{02}=2, x_{03}=y_{01}=0, a_{0}=3, a_{1}=-a_{3}=-1, \quad B=1 / 2, K_{1}=1 / 5, a_{2}=2$, we can obtain the following solution for the (2+1)-dimensional GBK system. Fig. (10) show the evolution of their interaction. We can find from the Fig.(10) that the two structures are fused to one structure which shape magnitude is not the summation of their ones after the head-on collision of them and it appears as the time increasing annihilation of the fused structure. In addition to the fusion and annihilation of the above mentioned structures with the increasing of time, we can also find in the Fig.(11), the fission of two structures into three and annihilation of the obtained structures after the collision. We take $f$ and $g$ as (36) with the same values of the above parameters except $k_{1}=1 / 8, \omega_{1}=\omega_{2}=-3, \omega_{3}=-2$. 
In other to study the interaction of the other kinds of the localized coherent structures, we choose different functions of $f$ and $g$. For instance, Fig.(12) shows the evolution of four localized structures with fusion, fission and annihilation after many collisions for the field $G$, second equation in system (35) for the following choice

$$
\begin{aligned}
& f=1+\exp \left\{\left[-\frac{4}{5}(x-2 t)^{2}+25\right]\right\}+\exp \left\{\left[-\frac{6}{5}(x+2 t)^{2}+25\right]\right\}, \\
& g=1+\exp \left\{(y-t)^{2}\right\} \exp \left(-K_{1} y\right)
\end{aligned}
$$

with $a_{0}=3, a_{1}=-1, a_{2}=2, a_{3}=1, K_{1}=1 / 5$.

The following choice of $f$ and $g$ leads to the evolution of the two structures in Fig.(13)

which enter into a collision and are subject to annihilation as time increasing,

$$
\begin{aligned}
& f=1-\tanh (t-2 x)-\tanh (t-x), \\
& g=1+\exp \left(-K_{1} y\right) \operatorname{sech}(2(y-t)),
\end{aligned}
$$

with $a_{0}=3, a_{1}=-1, a_{2}=2, a_{3}=1, K_{1}=1 / 5$.

When the following choice is done

$$
\begin{aligned}
& f=1+\operatorname{sech}(t-2 x)+\operatorname{sech}(t-x), \\
& g=1+\exp \left(-K_{1} y\right) \operatorname{sech}(2(y-t)),
\end{aligned}
$$

with $a_{0}=3, a_{1}=-1, a_{2}=2, a_{3}=1, K_{1}=1 / 5$. Then, the Fig.(14) is obtained.
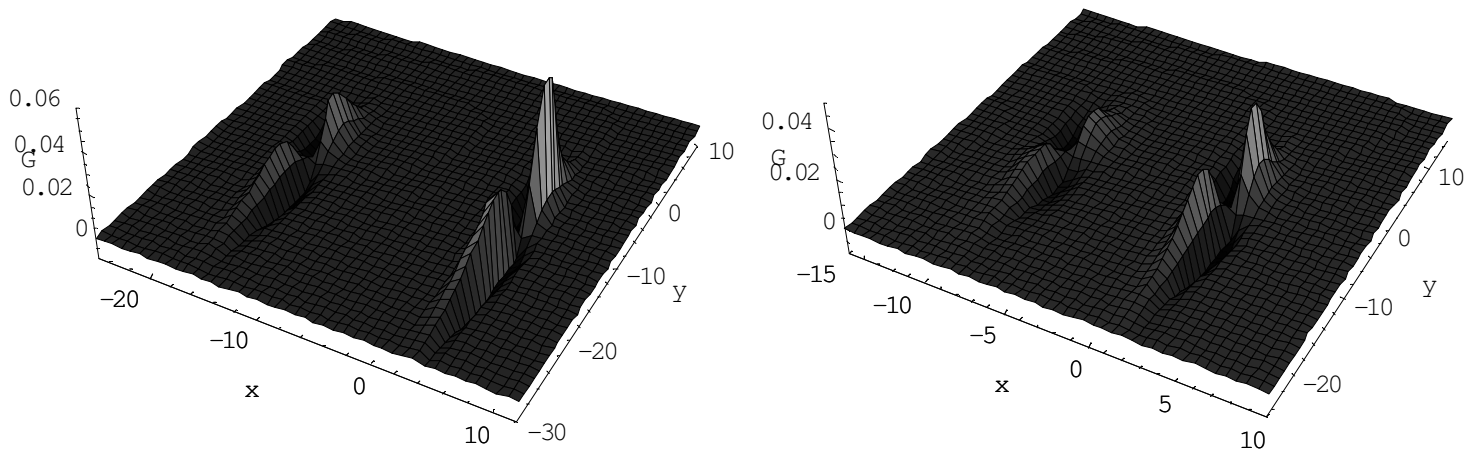
$t=-10$
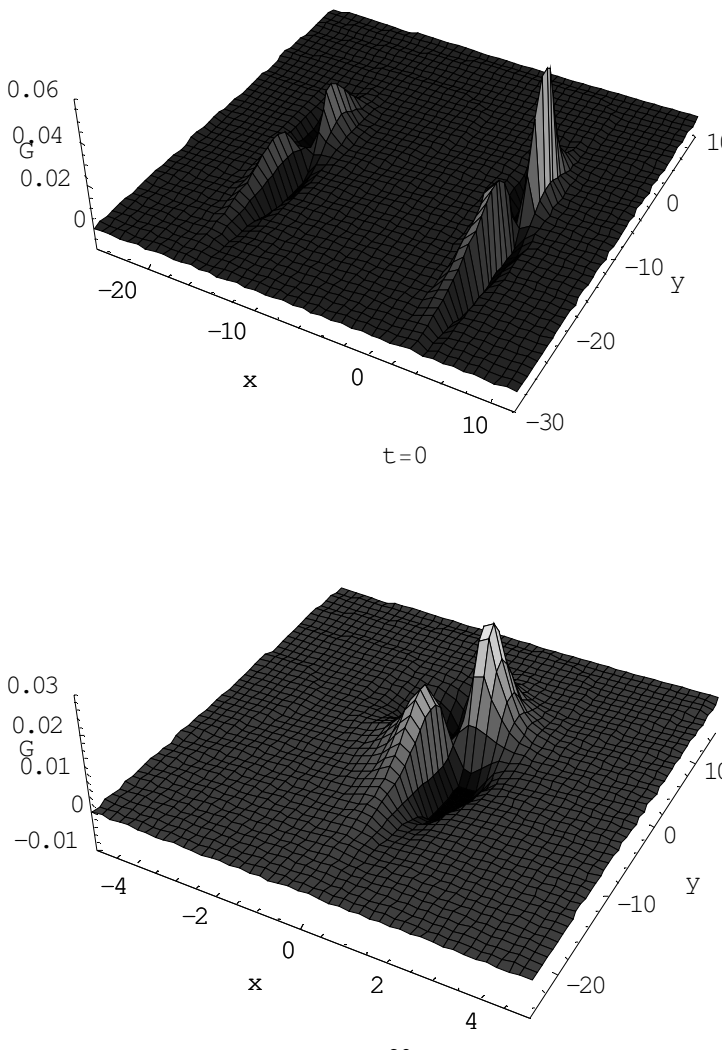

$t=20$

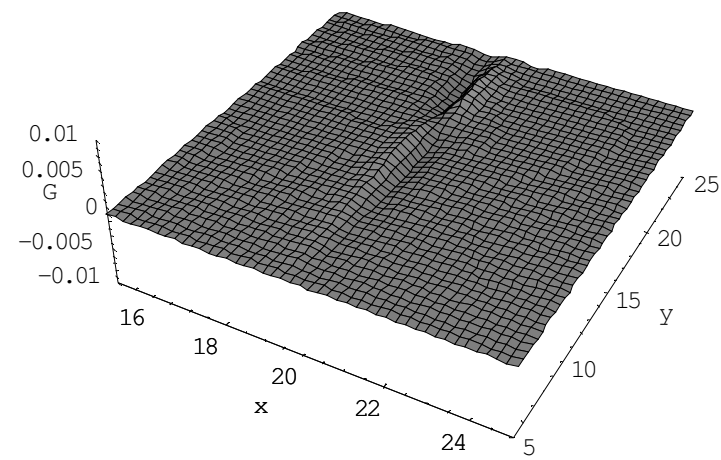

$t=-5$
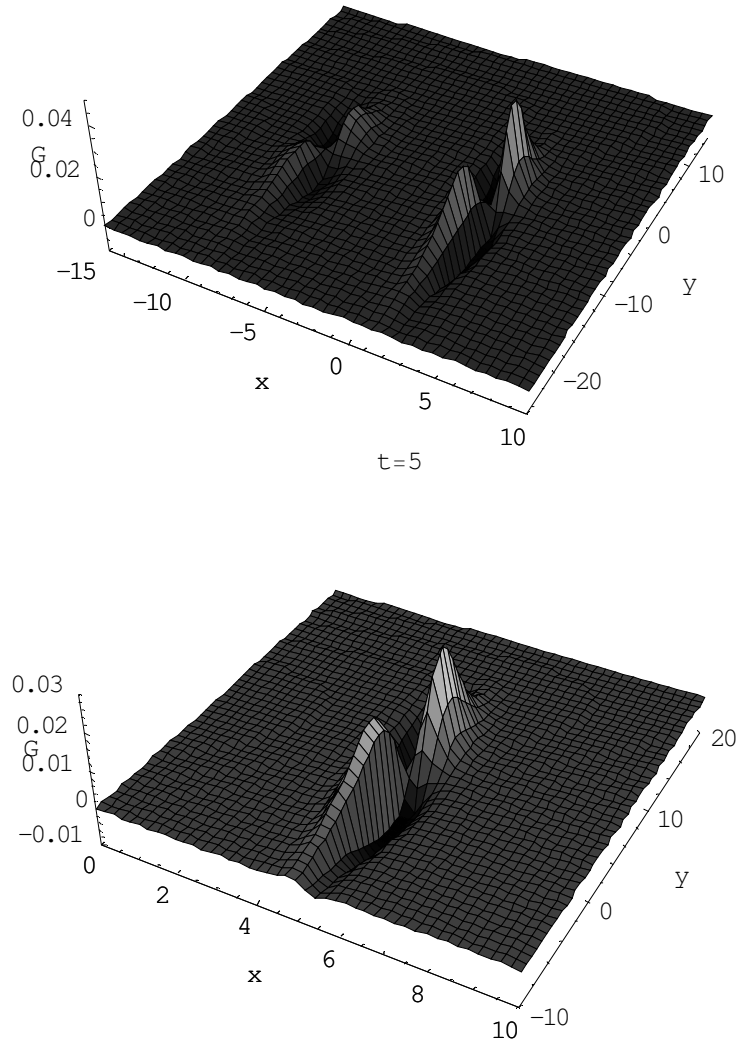

Fig.10. The evolution of two localized structure solutions Eq.(36) (first case). 

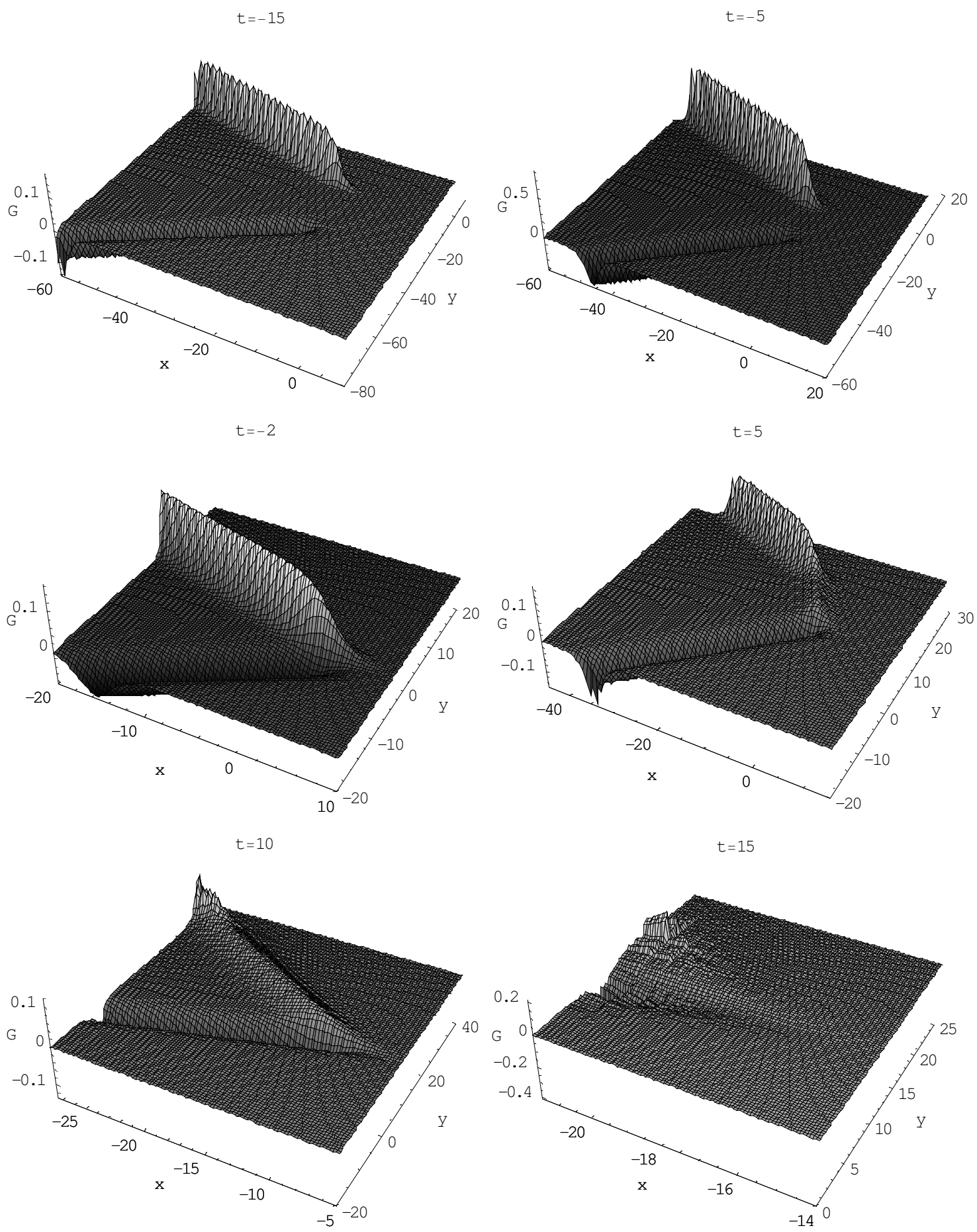

Fig.11. The evolution of two localized structure solutions Eq.(36) (second case). 




$t=1$



$t=10$
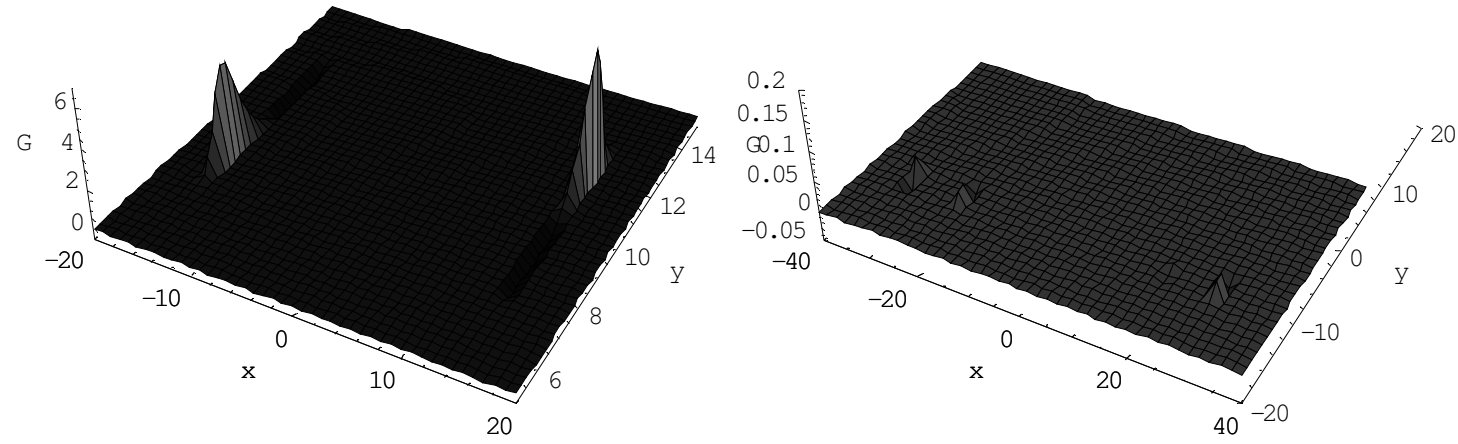

Fig.12. The evolution of four localized structure solutions Eq.(37) 

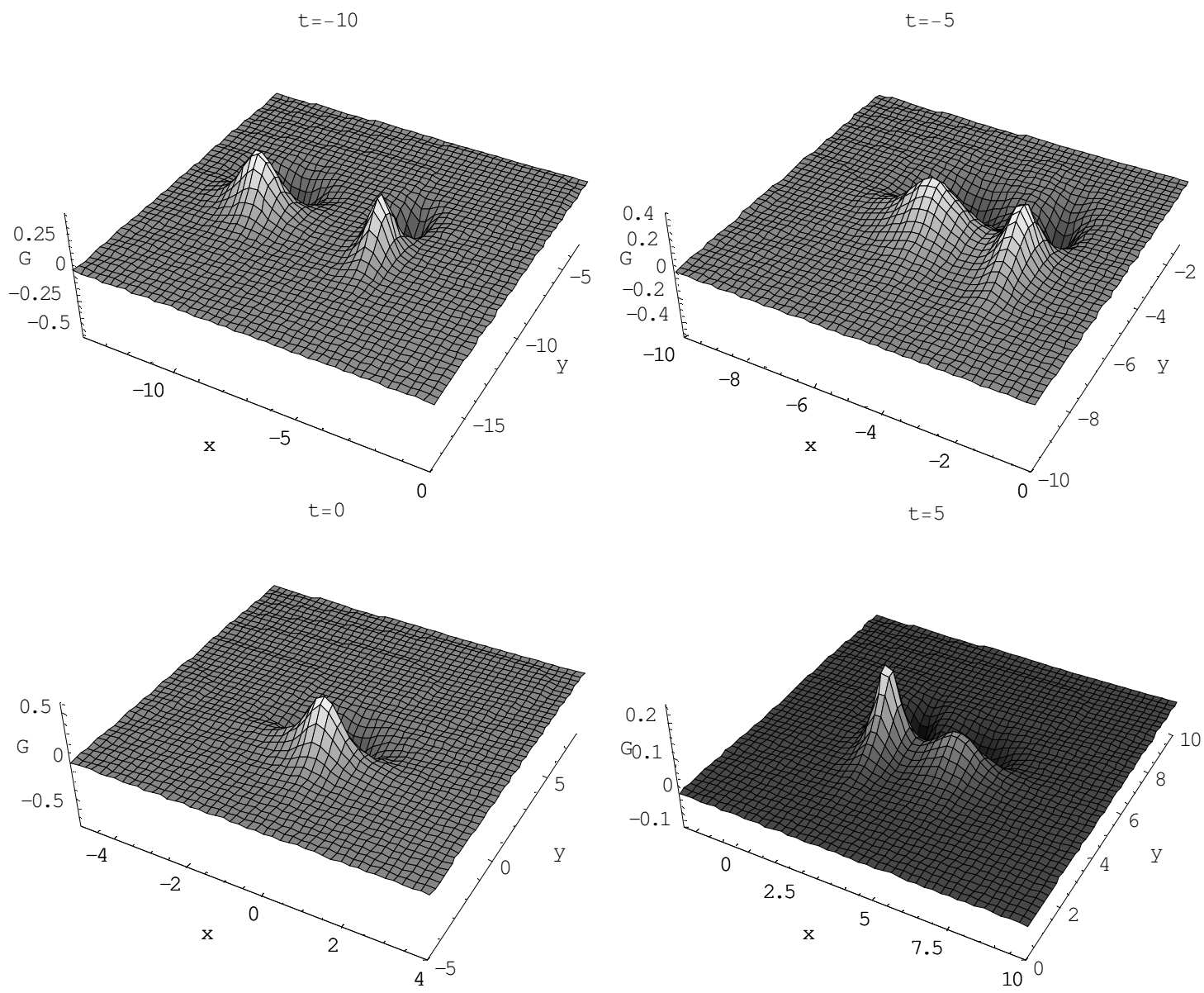

$t=10$

$t=18$
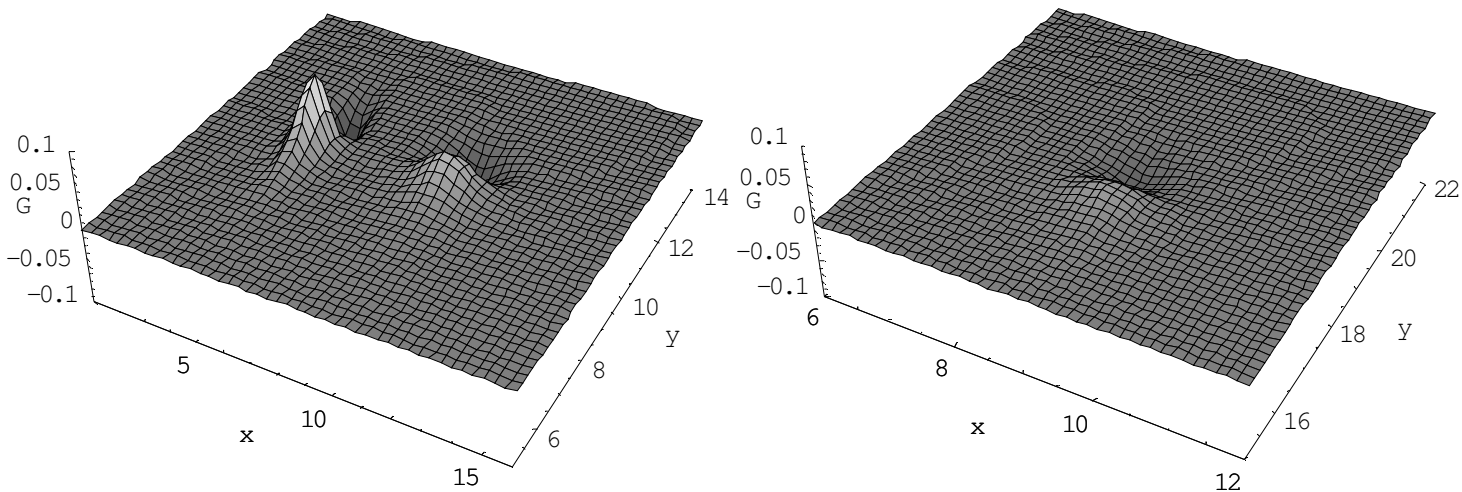

Fig.13. The evolution of two localized structure solutions Eq.(38). 

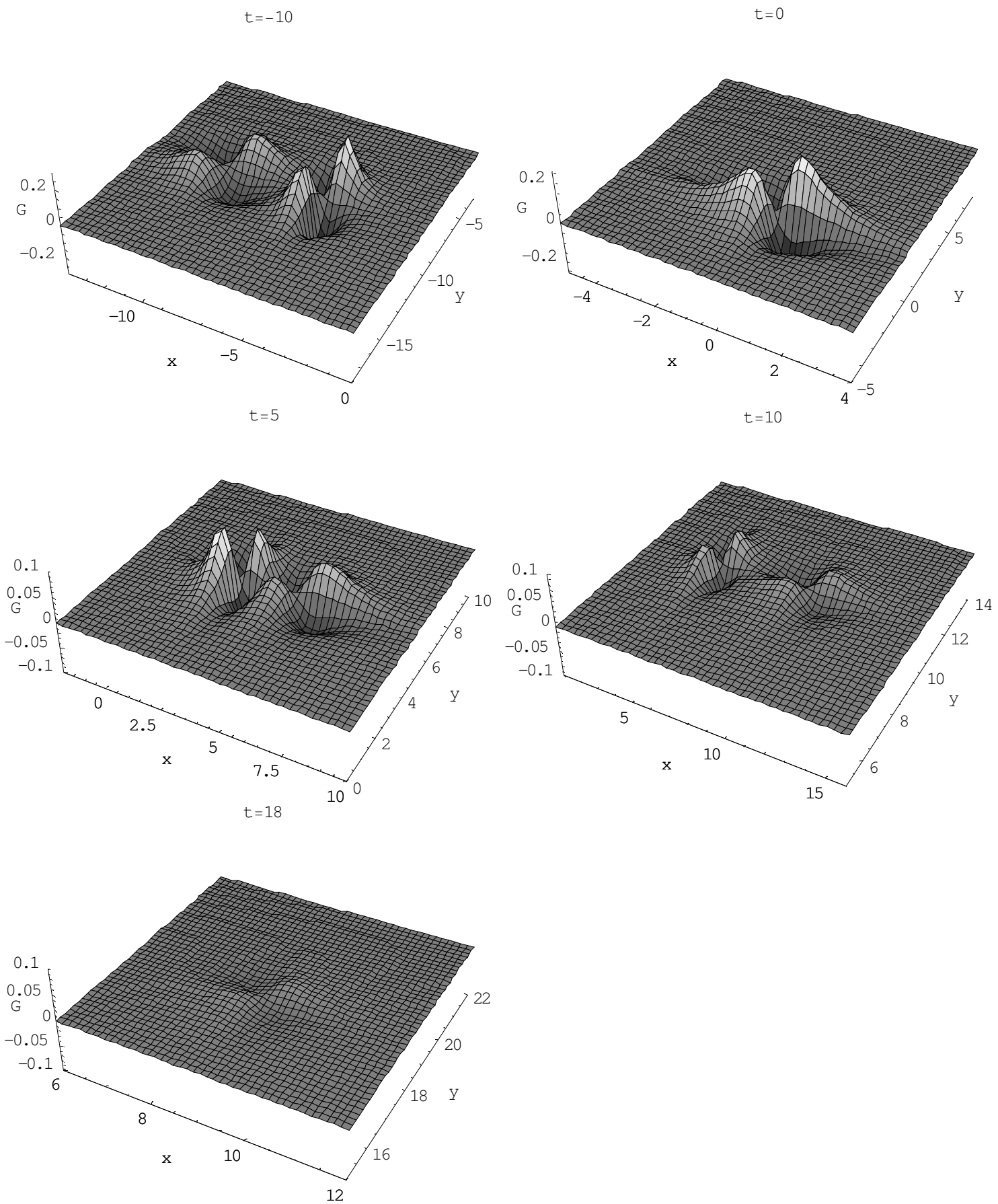

Fig.14. The evolution of two localized structure solutions Eq.(39).

The parameter $K_{1}$ rules the annihilation in the system. When $K_{1}$ is null there is no more annihilation in the system. Let us choose one of the above mentioned structures for example the first case of the solution (36) to illustrate this fact 


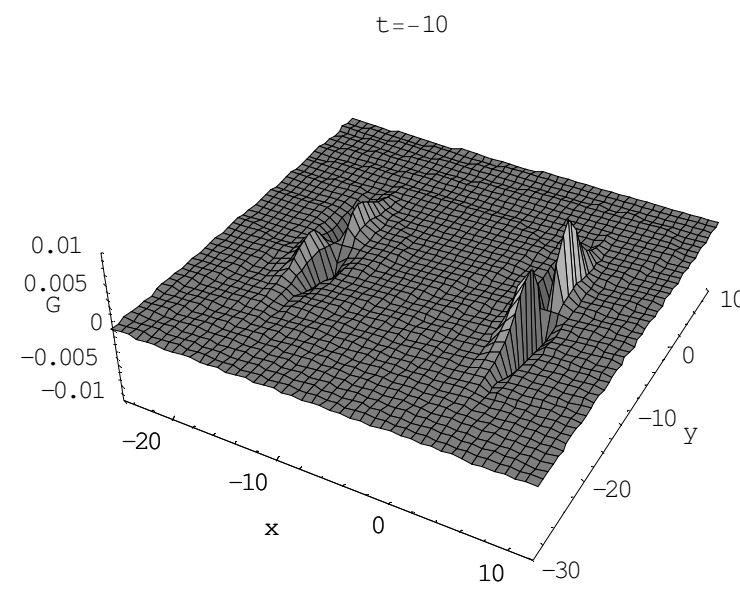

$\mathrm{t}=0$

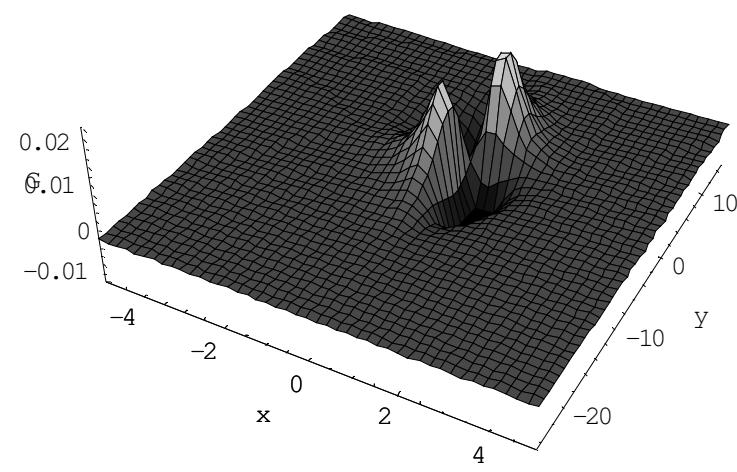

$t=20$

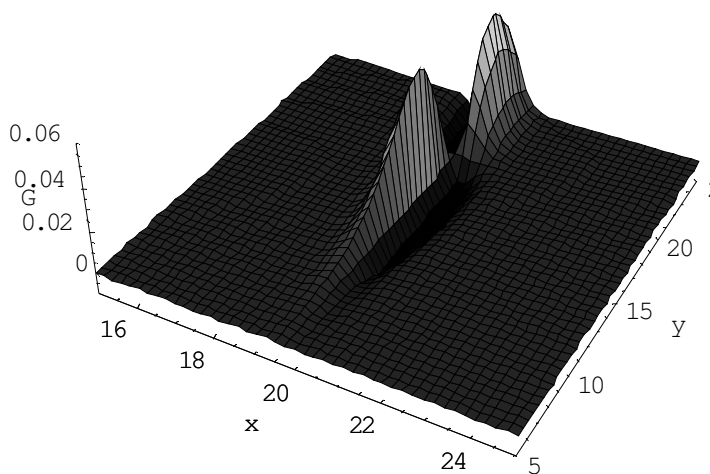

Fig.15. The evolution of two solitonic-excitations fusion Solution (36) (first case) with $K_{1}=0$.

\section{Conclusion}

Based on the WTC truncation method and the general variable separation approach (GVSA), we have first found a general solution including three arbitrary functions for the $(2+1)$-dimensional simplified generalized Broer-Kaup (GBK) system. 
A class of double periodic wave solutions is obtained by selecting these arbitrary functions appropriately. The interaction properties of the periodic waves are numerically studied and found to be non-elastic. Limit cases are considered and some new localized coherent structures are obtained, the interaction properties of these solutions reveal that some of them are completely elastic and some are non completely elastic.

Secondly, starting from the (2+1)-dimensional GBK system and using the variable separation approach (VSA), we obtained the excitations (30) which is universal formula for some (2+1)-dimensional physical models. Then, we have constructed by selecting the two arbitrary functions appropriately a rich variety of new coherent structures. For the localized solutions found here, it occurred that their interactions lead to new physical properties like fusion, fission, or both and finally the mutual annihilation of these solutions. It is clear that if a system exhibits annihilation of localized solutions the solutions are not solitons, since annihilation does not exist for the interactions of solitons. The annihilation in this model has found to be rule by the parameter $K_{1}$, when this parameter is taken to be zero, the annihilation disappears in this system and the above mentioned structures recover the solitonic structure properties.

\section{Acknowledgements}

Yomba is grateful to Professor George Sell of School of Mathematics of Minneapolis for valuable discussions. He will like to thank Profs. Douglas Arnold, Debra Lewis and Pamela Cook of the Institute for Mathematics and its Applications of Minneapolis for their warm-hearted help. His research was supported in part by the Institute for Mathematics and its Applications with funds provided by National Science Foundation.

Peng research is supported by the Postdoctoral Science Foundation of China

\section{References}

[1] Zhang SL, Wu B, Lou SY. Phys Lett A 2002;300:40.

[2] Broer LJF. Appl Sci Res 1975;31:377.

[3] Kaup DJ. Prog Theor Phys 1975;54:396.

[ 4] Huang DJ, Zhang HQ. Chaos, Solions \& Fractals 2005;23:601.

[5] Alber MS, Fedorov YN. Inverse Probl 2001;17:1017.

[6] Previato E. Duke Math J 1985;52:329.

[7] Porubov AV, Parker DF. Wave Motion 1999;29:97.

[ 8] Zhou RG. J Math Phys 1997;38:2535.

[ 9] Lou SY. Phys Lett A 2000;277:94. Lou SY. Phys Scr 2000;65:7. 
[10] Lou SY, Lu JZ. J Phys A Math Gen 1996;29:4029.

[11] Tang XY, Chen CL, Lou SY. J Phys A Math Gen 2002;35:293.

[12] Lou SY. J Phys A Math Gen 2002;35:10619.

[13] Lou SY, Tang XY, Chen CL, Lin J, Qian XM, Zhang SL. Mod Phys Lett B 2002;16:1075.

[14] Zhang J, Huang W, Zheng C. Acta Phys Sin 2002; 51:2676.

[15] Lou SY, Chen CL, Tang XY. J Math Phys 2002; 43:4078.

[16] Tang XY, Lou SY. nlin.SI/0210009.

[17] Tang XY, Lou SY. Chin Phys Lett. 2003;20:1075.

[18] Peng YZ. J Phys Soc Jpn 2005;74:287.

[19] Peng YZ. Phys Lett A 2005;337:55.

[20] Fan EG. Chaos, Solitons \& Fractals 2003;15:567.

[21] Peng YZ. J Phys Soc Jpn 2003;72:1356.

[22] Yomba E. Chaos, Solitons \& Fractals 2004;21:209.

[23] Chow KW. J Phys Soc Jpn 2000;69:1313. Chow KW. Phys Lett A 2004;326:404.

[24] Abramowitz M, Stegun IA. Handbook of Mathematical Functions Dover, New York, 1972.

[25] Weiss J, Tabor M, Carnevale G. J Math Phys 1983;24:522. 